\title{
BTB-1, ein niedermolekularer Inhibitor des mitotischen Motorproteins Kif18A**
}

Mario Catarinella, Tamara Grüner, Tobias Strittmatter, Andreas Marx und Thomas U. Mayer*

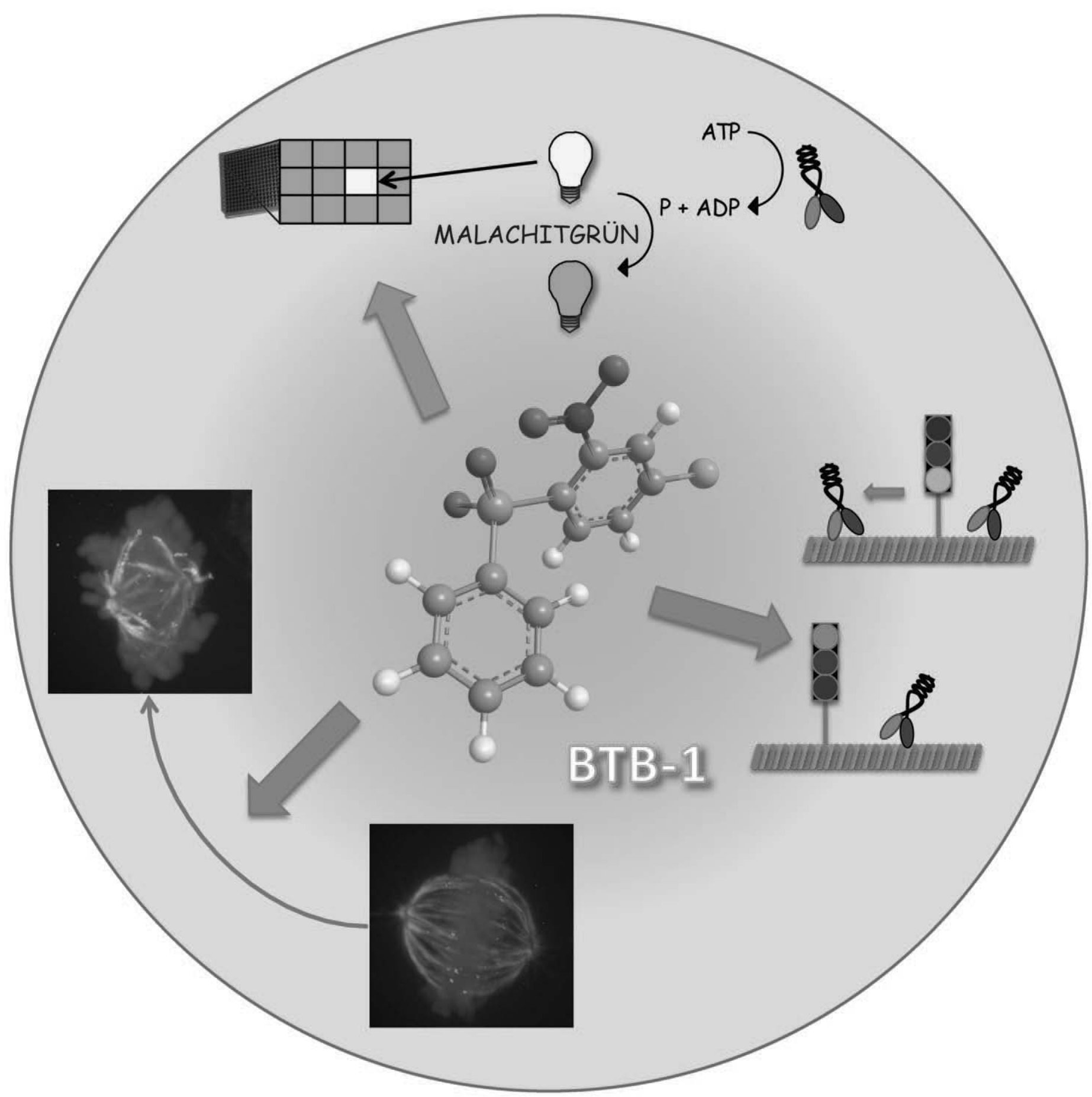


Das Überleben und die Entwicklung eines jeden Organismus hängen von der exakten Aufteilung seines Genoms während der Zellteilung ab. Bei höher entwickelten Lebewesen können Fehler in diesem Prozess zu schwerwiegenden Entwicklungsstörungen und Krebs führen. Im Zentrum der fehlerfreien Aufteilung des Genoms steht die mitotische Spindel, bestehend aus dynamischen Mikrotubuli (Mts). ${ }^{[1]}$ Die Form und die Funktion der mitotischen Spindel hängen von der koordinierten Aktivität verschiedener Kinesine ab, molekularer Motorproteine, die als mikrotubulistimulierte ATPasen chemische Energie in mechanische Kraft umwandeln. Kürzlich identifizierten wir Kif18A, ein Kinesin-8-Mitglied, als eine zentrale Komponente für die korrekte Ausrichtung der Chromosomen am Spindeläquator. ${ }^{[2]}$ Zudem zeigten In-vitroAnalysen, dass sich Kif18A von allen bekannten Kinesinen durch seine Doppelfunktionalität - die Motilität und die Depolymeraseaktivität - unterscheidet. ${ }^{[2-4]}$

Wegen der schnellen und oft reversiblen Wirkmechanismen niedermolekularer Verbindungen eignen sich diese ideal zur Aufklärung der Funktionen und Mechanismen von Proteinen. Angesichts der komplexen Enzymeigenschaften von Kif18A entschlossen wir uns für ein Screening nach niedermolekularen Kif18A-Inhibitoren. Hier berichten wir über die Entdeckung von BTB-1 (Abbildung 1 a), dem ersten niedermolekularen Inhibitor von Kif18A. Wir zeigen, dass BTB-1 offensichtlich die ATPase-Aktivität von Kif18A inhibiert $\left(\mathrm{IC}_{50}=1.69 \mu \mathrm{M}\right)$, aber nicht diejenige weiterer getesteter, für die Mitose essenzieller Kinesine. BTB-1 blockiert die Motilität von Kif18A reversibel. Bemerkenswert ist, dass BTB-1 Kif18A in einem Adenosintriphosphat(ATP)-kompetitiven und Mikrotubuli-unkompetitiven Modus hemmt und den Mitoseablauf in Zellen verlangsamt.

Zur Identifizierung von Kif18A-Inhibitoren durchmusterten wir eine Bibliothek aus 9000 unterschiedlichen niedermolekularen Molekülen nach Substanzen, die die ATPase-Aktivität der rekombinanten Motordomäne von Kif18A (GST-Kif18A ${ }^{\text {motor }}$; GST = Glutathion-S-Transferase) in vitro hemmen. Die Freisetzung von Phosphat wurde mithilfe eines auf Malachitgrün basierenden Assays untersucht und so die Aktivität von GST-Kif18A ${ }^{\text {motor }}$ ausgelesen (siehe Hintergrundinformationen). Die Verbindungen wurden im Duplikat bei einer Konzentration von $50 \mu \mathrm{M}$ durchmustert und als Treffer berücksichtigt, wenn sie die Kif18A ${ }^{\text {motor }}$-vermittelte ATP-Hydrolyse zu mehr als $65 \%$ inhibierten. Von den vier identifizierten Treffern war BTB-1 (Abbildung 1a)

[*] M. Catarinella, T. Strittmatter, Prof. Dr. A. Marx, Prof. Dr. T. U. Mayer Konstanz Research School Chemical Biology

Universität Konstanz, 78457 Konstanz (Deutschland)

Fax: (+ 49) 7531-88-3707

E-Mail: Thomas.u.Mayer@uni-konstanz.de

Homepage: http://www.uni-konstanz.de/thomasmayer/

T. Grüner

London Research Institute, Lincoln's Inn Fields Laboratories WC2 A3PX London (Großbritannien)

[***] Für die Finanzierung danken wir dem DAAD und den Marie Curie Actions 6. Zudem danken wir Prof. Dr. W. Hofer und den Mitgliedern der Arbeitsgruppe Mayer für die Diskussionen.

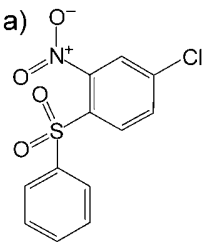

a)

b)

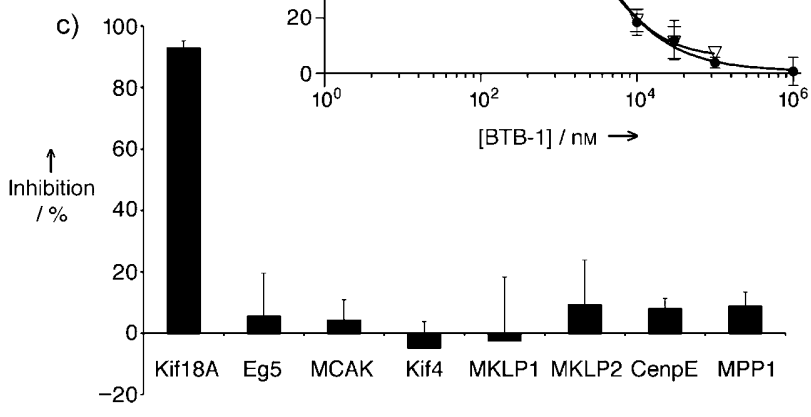

Abbildung 1. a) Strukturformel von BTB-1. b) Steigende Konzentrationen an BTB-1 (•) und resynthesiertem BTB-1 $(\nabla)$ wurden genutzt, um den $\mathrm{IC}_{50}$-Wert zu bestimmen. c) Der Inhibitoreffekt von $100 \mu \mathrm{M}$ BTB-1, getestet mit einem enzymgekoppelten Assay an verschiedenen rekombinanten Kinesinen (siehe Hintergrundinformationen). Die Mittelwerte dreier unabhängiger Experimente mit Standardabweichungen sind gezeigt.

der stärkste und selektivste und wurde für weitere Analysen ausgewählt. Den $\mathrm{IC}_{50}$-Wert bestimmten wir, indem wir einen enzymgekoppelten Assay verwendeten, wobei die Geschwindigkeit der ATP-Hydrolyse durch His-Kif18A ${ }^{\text {motor }}$ in Gegenwart von Mts und steigenden Konzentrationen an BTB-1 oder DMSO als Kontrolle verfolgt wurde (siehe Hintergrundinformationen). BTB-1 inhibierte mit einem $\mathrm{IC}_{50}$-Wert von $1.69 \mu \mathrm{M}$ die Mt-stimulierte ATPase-Aktivität von His-Kif18A ${ }^{\text {motor }}$ (Abbildung 1b). BTB-1 ist damit der erste bekannte Inhibitor von Kif18A, einem von der Hefe bis zum Menschen konservierten Schlüsselenzym der Chromosomenkongression. Um die chemische Identität von BTB-1 $\mathrm{zu}$ bestätigen, wurde das Molekül resynthesiert (siehe Hintergrundinformationen). Das resynthesierte BTB-1 hemmte His-Kif18A ${ }^{\text {motor }}$ gleich stark $\left(\mathrm{IC}_{50}=1.86 \mu \mathrm{M}\right.$; Abbildung $\left.1 \mathrm{~b}\right)$. Als nächstes befassten wir uns mit der Selektivität von BTB-1. Entsprechende Studien ergaben, dass $100 \mu \mathrm{M}$ BTB-1 keines der weiteren getesteten mitotischen Kinesine signifikant inhibierte (Abbildung 1c).

Um Einblicke in den Bindungsmodus von BTB-1 zu erhalten, analysierten wir zuerst, ob BTB-1 reversibel an Kif18A bindet. Zu diesem Zweck entwickelten wir einen Invitro-Mt-Gleitassay unter Verwendung einer Durchströmkammer, die einen schnellen Austausch der Reaktionslösung ermöglichte. Die Bewegung von Mts wurde durch Echtzeitmikroskopie aufgenommen und mit einem Kymographen angezeigt, in dem die Position eines individuellen Mt (horizontale Achse) gegen die Funktion der Zeit (vertikale Achse) aufgetragen wurde (Abbildung 2). In Einklang mit der Literatur bewegte das auf einer Glasplatte immobilisierte, rekombinante Volllängen-Kif18A (His-Kif18A ${ }^{\mathrm{FL}}$ ) in Gegenwart von DMSO die fluoreszenzmarkierten Mikrotubuli mit einer Geschwindigkeit von $(0.036 \pm 0.015) \mu \mathrm{m} \mathrm{min}^{-1}$ (Abbildung 2 b,c und Film S1 in den Hintergrundinformationen). ${ }^{[2]}$ 
a)

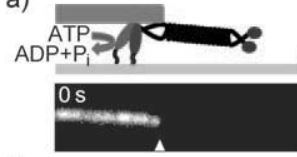

b)

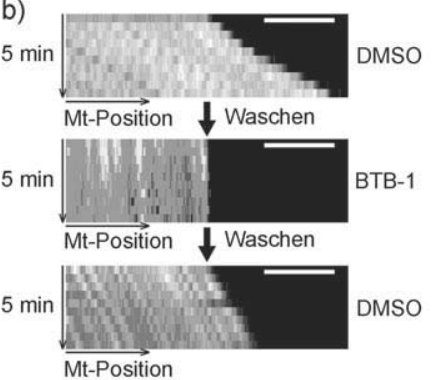

d)

e)

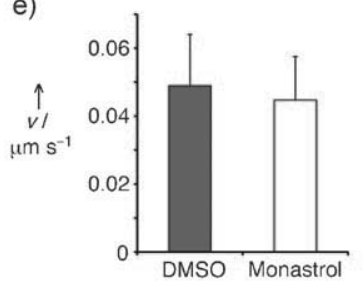

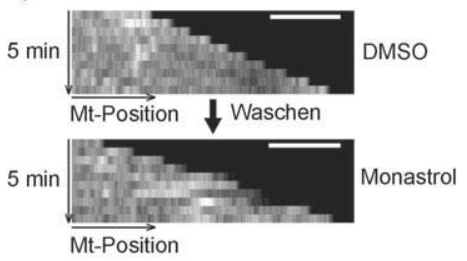

Abbildung 2. a) Rekombinantes His-Kif18 $\mathrm{A}^{\mathrm{FL}}$ wurde auf einer Glasoberfläche adsorbiert und mit rhodaminmarkierten Mts inkubiert. Unter ATP-Verbrauch kann Kif18A Mikrotubuli bewegen. Oben: schematische Darstellung des Assays ( $r o t=M t$, blau-schwarze Struktur $=$ Kif18A, hellblau $=$ Glasoberfläche; $A D P=$ Adenosindiphosphat, $\mathrm{P}_{\mathrm{i}}=$ Phosphat). Unten: Fluoreszenzaufnahmen der Kif18A-vermittelten Mikrotubulibewegung (Pfeile zeigen die Position der Mikotubulusspitze zum jeweiligen Zeitpunkt; Maßstab $=5 \mu \mathrm{m}$ ). b) Repräsentative Kymographen eines Mt-Gleitassays, durchgeführt in Gegenwart von DMSO, nach der Spülung mit $100 \mu \mathrm{M}$ BTB-1 und nach dem Auswaschen von BTB-1 (siehe Text sowie Filme S1-S3 in den Hintergrundinformationen). c) Quantifizierung der Mt-Motilität (10 Mts, Mittelwerte dreier unabhängiger Experimente mit Standardabweichungen sind gezeigt). d) Repräsentative Kymographen einer His-Kif18 $\mathrm{A}^{\mathrm{FL}}$-vermittelten Bewegung eines $\mathrm{Mt}$ in Gegenwart von DMSO oder $100 \mu \mathrm{M}$ Monastrol (siehe Film S4 in den Hintergrundinformationen). e) Quantifizierung der MtMotilität (10 Mts, Mittelwerte dreier unabhängiger Experimente mit Standardabweichungen sind gezeigt).

Einspülen von $100 \mu \mathrm{M}$ BTB-1 hob nahezu die gesamte HisKif18A ${ }^{\mathrm{FL}}$-abhängige Mt-Motilität auf (Abbildung 2b,c und Film S2 in den Hintergrundinformationen). Dieser Effekt war spezifisch, denn $100 \mu \mathrm{M}$ an Monastrol, einem selektiven Inhibitor des mitotischen Kinesins Eg5, ${ }^{[5]}$ beeinflussten die His-Kif18A $\mathrm{A}^{\mathrm{FL}}$-abhängige Motilität nicht (Abbildung $2 \mathrm{~d}$,e und Film S4 in den Hintergrundinformationen). Nach dem Auswaschen von BTB-1 erlangte His-Kif18A ${ }^{\mathrm{FL}}$ einen Großteil seiner Aktivität zurück und bewegte Mts bei $(0.027 \pm$ $0.013) \mu \mathrm{min}^{-1}$ (Abbildung 2 b,c und Film S3 in den Hintergrundinformationen). Demzufolge ist BTB-1 ein reversibler Inhibitor von Kif18A.

Wenn Kinesine entlang der Mikrotubuli laufen, hydrolysieren sie ATP unter dessen Verwendung als Bona-FideSubstrat, d.h. als echtes Substrat, und der Mikrotubuli als Pseudosubstrate, die nicht enzymatisch umgesetzt werden. Zuerst erforschten wir, ob BTB-1 mit ATP um die Kif18ABindung konkurriert. Dafür ermittelten wir die Geschwindigkeit der Mt-stimulierten ATP-Hydrolyse von His-
Kif18A ${ }^{\text {motor }}$ bei einer Mikrotubulisättigungskonzentration und bei variierenden Konzentrationen an ATP und BTB-1 und glichen jeden Datensatz der Michaelis-Menten-Gleichung an (siehe Hintergrundinformationen). Wie aus Abbildung 3 a ableitbar, steigerte BTB-1 den $K_{\mathrm{m}}$-Wert für ATP, hatte jedoch keinen signifikanten Einfluss auf den $V_{\max }$-Wert. ATP $\gamma$ S, ein nicht hydrolysierbares, mit ATP konkurrierendes Analogon, beeinflusste die $K_{\mathrm{m}}$ - und $V_{\max }$-Werte auf ähnliche Weise (Abbildung 3b). Daraus kann geschlossen werden, dass BTB-1 Kif18A in einer ATP-kompetitiven Weise inhibiert.

Als nächstes untersuchten wir, wie Mikrotubuli die Wirkung von BTB-1 auf Kif18A beeinflussen. Dazu wurde die Geschwindigkeit der His-Kif18A ${ }^{\text {motor }}$-vermittelten ATP-Hydrolyse bei ATP-Sättigungskonzentration und bei variierenden Konzentrationen von Mts und BTB-1 quantifiziert (Abbildung 3c). BTB-1 beeinflusste sowohl den $K_{1 / 2}$-Wert für Mts (bei Pseudosubstraten wird der Ausdruck $K_{1 / 2}$ anstelle von $K_{\mathrm{m}}$ verwendet) als auch den $V_{\max }$-Wert, was darauf schließen lässt, dass BTB-1 in einer nicht-kompetitiven, unkompetitiven oder gemischt-kompetitiven Weise wirkt.

Eine genaue Analyse der Michaelis-Menten-Kinetiken identifizierte den unkompetitiven Wirkmechanismus als die wahrscheinlichste Wirkungsweise. Dies würde bedeuten, dass BTB-1 nur an den Komplex aus Kif18A und Mikrotubuli binden kann. Würde dies zutreffen, ließe sich vorhersagen, dass BTB-1 nicht die basale, mikrotubuliunabhängige ATPase-Aktivität von Kif18A inhibiert. Um dies zu testen, nutzten wir einen enzymgekoppelten Assay, um die basale ATPase-Aktivität des rekombinanten His-Kif18A ${ }^{\text {motor }}$ vor der Zugabe von DMSO/BTB-1 (Phase I), nach der Zugabe von DMSO/BTB-1 (Phase II) sowie die durch die Gegenwart von Mts stimulierte ATPase-Aktivität von His-Kif18A ${ }^{\text {motor }}$ in Gegenwart von DMSO oder BTB-1 (Phase III) zu messen. Um die niedrige Mt-unabhängige ATPase-Aktivität von Kif18A messen zu können, mussten wir hohe Konzentrationen an His-Kif18A ${ }^{\text {motor }}$ und BTB-1 verwenden. Vor der Zugabe von BTB-1 (blaue Linie) oder DMSO (rote Linie) hydrolysierte His-Kif18A ${ }^{\text {motor }}$ ATP bei einer Geschwindigkeit von ca. $0.12 \mathrm{~s}^{-1}$ (Abbildung 3d, Phase I). Die Hydrolyse von ATP wurde durch His-Kif18A ${ }^{\text {motor }}$ vermittelt, da in Abwesenheit des Motorproteins keine ATP-Hydrolyse gemessen werden konnte (Abbildung 3d, violette (DMSO) schwarze Linien (BTB-1)). Dabei beeinflusste die Zugabe von $100 \mu \mathrm{M}$ BTB-1 nicht die basale ATPase-Aktivität von His-Kif18A ${ }^{\text {motor }}$ in Abwesenheit von Mts (Abbildung 3d,f, Phase II: $0.12 \mathrm{~s}^{-1}$ (BTB-1) und $0.11 \mathrm{~s}^{-1}$ (DMSO)). Die vorübergehende $\mathrm{Zu}-$ nahme der Absorption nach Zugabe von BTB-1 stand nicht im Zusammenhang mit Kif18A, da der gleiche Effekt in der Probe ohne Motorprotein beobachtet wurde (Abbildung 3d, schwarze Linie, Phase II). Wichtig war, dass BTB-1 die Mtstimulierte ATPase-Aktivität von His-Kif18A ${ }^{\text {motor }}$ signifikant inhibierte (Abbildung 3d,f, Phase III: $0.13 \mathrm{~s}^{-1}$ (BTB-1) und $0.3 \mathrm{~s}^{-1}$ (DMSO)), was unser Modell bestätigte, demzufolge die Inhibitoraktivität von BTB-1 von der Komplexbildung von Kif18A und Mts abhängig ist. Monastrol inhibierte, in Übereinstimmung mit früheren Publikationen, ${ }^{[6]}$ sowohl die basale als auch die Mt-stimulierte ATPase-Aktivität von Eg5 (Abbildung $3 \mathrm{e}, \mathrm{g}$ ). Insgesamt lassen diese Daten darauf 
a)
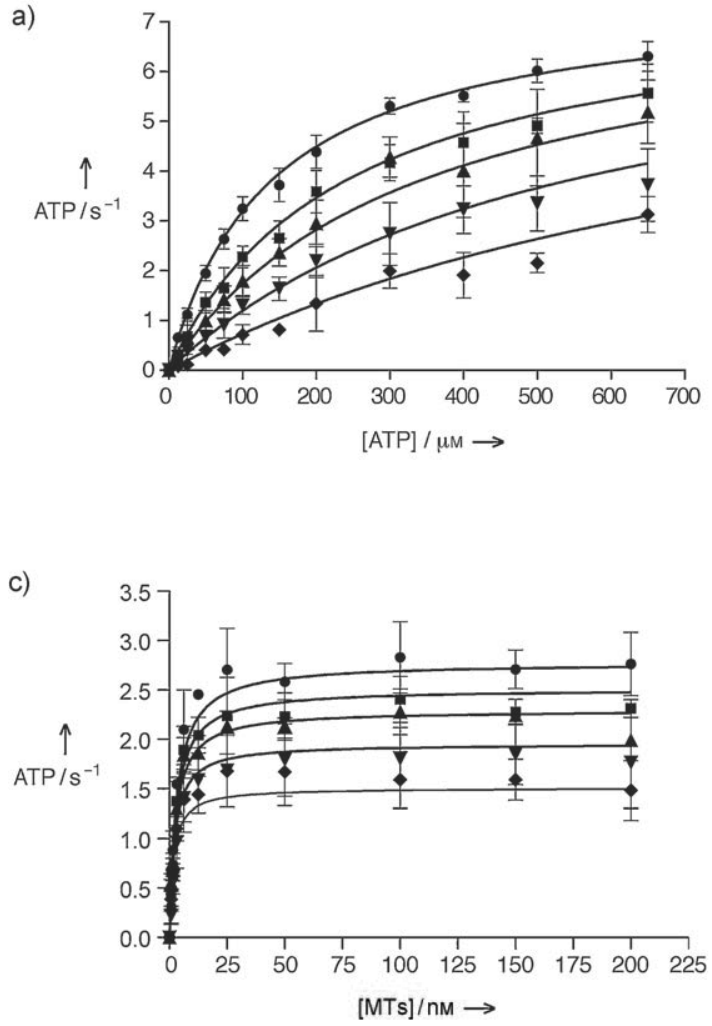

e)

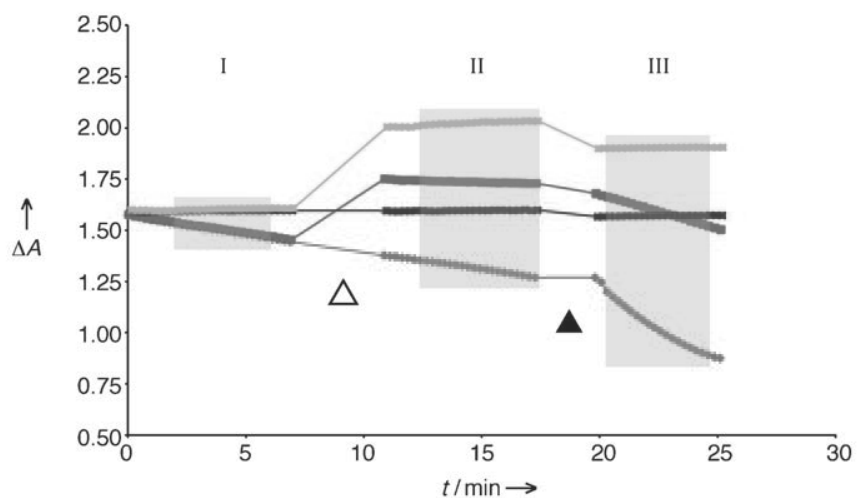

b)

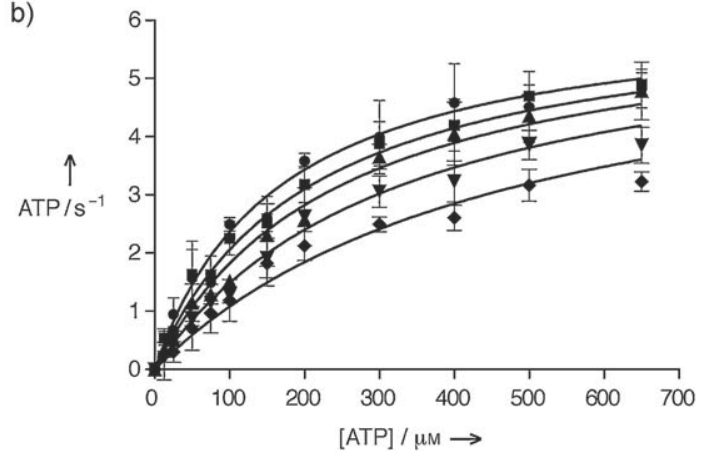

d)

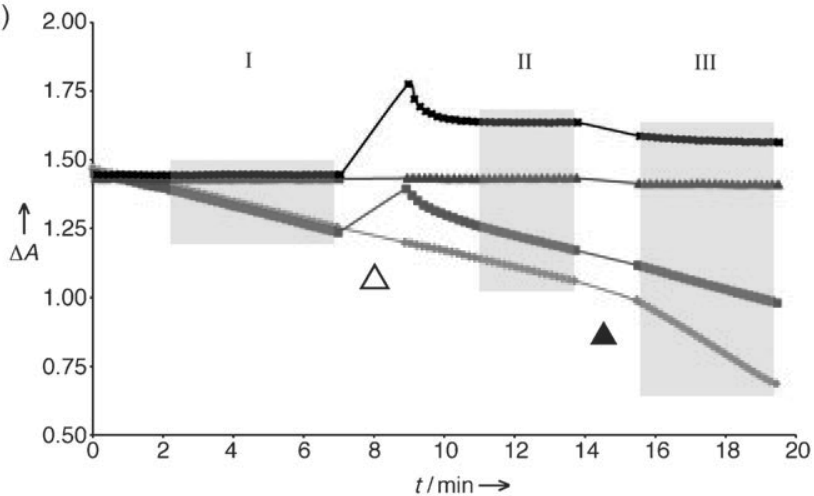

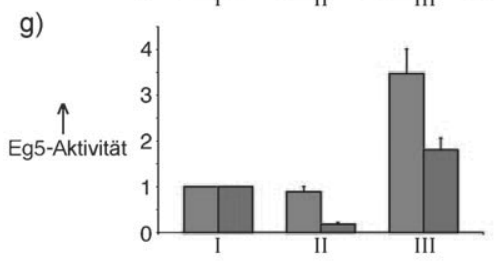

Abbildung 3. a) Die Aktivität von His-Kif1 $8 \mathrm{~A}^{\text {motor }}$ bei steigenden ATP-Konzentrationen wurde überwacht in Gegenwart von $3 \mu \mathrm{M}$ Mts und steigenden BTB-1-Konzentrationen $(\boldsymbol{=}=0.21 \mu \mathrm{M}, \boldsymbol{\nabla}=0.42 \mu \mathrm{M}, \boldsymbol{\Delta}=0.85 \mu \mathrm{M}, \bullet=1.7 \mu \mathrm{M})$ oder DMSO als Kontrolle ( $\bullet$ ). b) Steigende ATP $\gamma \mathrm{S}-\mathrm{Konzentratio-}$

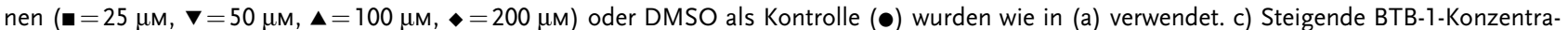
tionen $(\boldsymbol{\nabla}=0.21 \mu \mathrm{M}, \boldsymbol{\nabla}=0.42 \mu \mathrm{M}, \boldsymbol{\Delta}=0.85 \mu \mathrm{M}, \bullet=1.7 \mu \mathrm{M})$ oder DMSO als Kontrolle $(\bullet)$ wurden an His-Kif18A ${ }^{\text {motor }}$ bei $650 \mu \mathrm{M}$ ATP und variierenden Konzentrationen an Mts getestet. d) ATPase-Aktivität bei 705 nM His-Kif18A ${ }^{\text {motor }}$. Phase I: Basale ATPase-Aktivität vor Zugabe von DMSO (rote Linie) oder $100 \mu \mathrm{M}$ BTB-1 (blaue Linie). Phase II: Basale ATPase-Aktivität nach Zugabe von DMSO oder $100 \mu \mathrm{M}$ BTB-1. Phase III: Mt-stimulierte ATPase-Aktivität in Gegenwart von DMSO oder $100 \mu \mathrm{M}$ BTB-1. Violette Linie: DMSO-Kontrollreaktion ohne His-Kif18A ${ }^{\text {motor }}$. Schwarze Linie: BTB-1-DMSO-Kontrollreaktion ohne His-Kif18A motor. $\triangle$ : DMSO/BTB-1 Zugabe; $\mathbf{\Delta}$ : Zugabe von Mts; hellgraue Bereiche: Zeitpunkte, um in der jeweiligen Phase die ATP-Hydrolyserate zu berechnen. e) ATPase-Aktivität von $800 \mathrm{~nm} \mathrm{His-Eg5}{ }^{\text {motor }}$. Rote Linie: DMSO; dunkelgrüne Linie: 100 MM Monastrol; violette Linie: DMSO-Kontrolle ohne His-Eg $5^{\text {motor }}$; hellgrüne Linie: Monastrolkontrolle ohne His-Eg5 ${ }^{\text {motor }}$. f) Quantifizierung der HisKif18A $\mathrm{A}^{\text {motor }}$-Aktivität während Phase I, II und III, wie in (d) beschrieben; Mittelwerte dreier unabhängiger Experimente mit Standardabweichungen

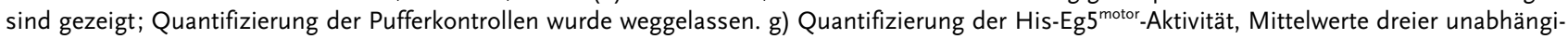
ger Experimente mit Standardabweichungen sind gezeigt; Quantifizierung der Pufferkontrollen wurde weggelassen.

schließen, dass BTB-1 das Enzym Kif18A ATP-kompetitiv und Mt-unkompetitiv inhibiert.

Zum Schluss testeten wir, ob BTB-1 den Mitoseablauf von HeLa-Zellen beeinflusst. Die RNA-Interferenz(RNAi)-vermittelte Depletion von Kif18A verursacht schwerwiegende
Defekte in der Spindelmorphologie, einhergehend mit Fehlern in der Chromosomenkongression, wodurch Zellen im frühen Stadium der Mitose arretieren. ${ }^{[2,7]}$ Bemerkenswert ist, dass mit BTB-1 behandelte HeLa-Zellen konzentrationsabhängig in der Mitose akkumulieren (Abbildung 4c). Im- 
a)

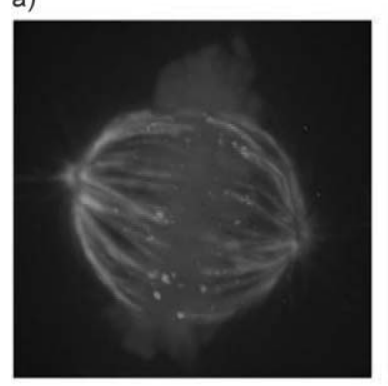

b)

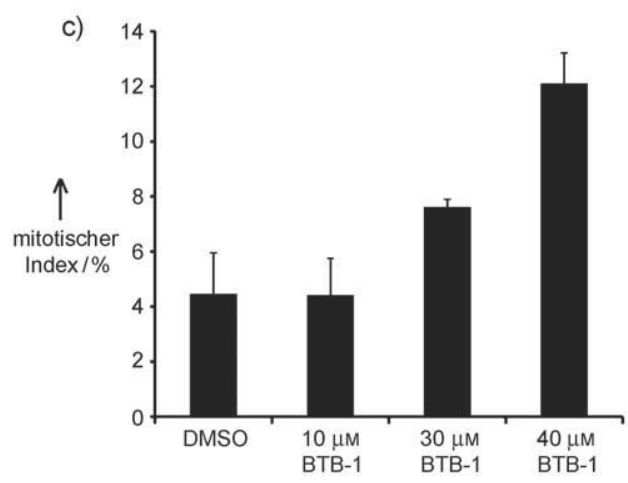

Abbildung 4. Immunfluoreszenzaufnahmen von HeLa-Zellen: $18 \mathrm{~h}$ behandelt mit a) DMSO oder b) $30 \mu \mathrm{M}$ BTB-1. Rot $=$ Kif18A; grün $=$ Mikrotubuli; blau $=$ DNA (Maßstab $=5 \mu \mathrm{m})$. c) Quantifizierung des mitotischen Index von HeLa-Zellen, behandelt mit DMSO oder steigenden BTB-1-Konzentrationen ( $\geq 180$ Zellen; Mittelwerte dreier unabhängiger Experimente mit Standardabweichungen sind gezeigt).

munfluoreszenzbilder zeigten, dass die Spindelstrukturen in mit BTB-1 behandelten Zellen stark beeinträchtigt waren (Abbildung 4b). Es konnten allerdings keine verlängerten Spindeln, wie sie bei der RNAi-vermittelten Depletion von Kif18A vorkommen, ${ }^{[2]}$ in mit BTB-1 behandelten Zellen detektiert werden. Durch die Doppelfunktionalität von Kif18A - es kann entlang den Mts laufen und deren Enden depolymerisieren - wird jedoch die Interpretation von durch unterschiedliche Ansätze verursachten Phänotypen erschwert, d.h., die Entfernung von Kif18A aus dem zellulären Kontext durch RNAi kann andere Auswirkungen haben als die Inhibition der ATPase-Aktivität von Kif18A durch BTB-1. Folglich sind weitere Studien notwendig, um Kif18A eindeutig als das relevante Zielprotein in Zellen zu bestätigen.

Wir haben hier von der Entdeckung von BTB-1 berichtet, dem ersten Inhibitor von Kif18A, der durch ein Protein-basiertes, revers-chemisch-genetisches Screening identifiziert wurde. Detaillierte enzymatische Studien zeigten, dass BTB-1 ein starker Inhibitor von Kif18A ist $\left(\mathrm{IC}_{50}=1.69 \mu \mathrm{M}\right)$, der reversibel in einem ATP-kompetitiven und Mt-unkompetitiven Modus wirkt, d.h., BTB-1 konkurriert mit ATP nur um die Bindung an Kif18A, wenn das Motorprotein an sein Pseudosubstrat, den Mikrotubulus, gebunden ist. Dieses außergewöhnliche Merkmal von BTB-1, kombiniert mit dessen schnellem und reversiblem Inhibitionsmodus, macht diesen
Inhibitor zu einem leistungsfähigen Hilfsmittel für die Aufklärung der mechanochemischen Eigenschaften von Kif18A, einem Kinesin, das Mikrotubulimotilität und Depolymeraseaktivität in sich vereint. Der chemisch-biologische Ansatz hat uns daher ein neues Hilfsmittel in die Hand gegeben, um die Schlüsselproteine der Chromosomsegregation in Säugetierzellen zu studieren. Beispiele wie Inhibitoren von mitotischem Kinesin Eg5, ${ }^{[5,8-10]}$ nicht-muskulärem Myosin II, ${ }^{[1]}$ Polo-ähnlichen $(\mathrm{Plk1})^{[12-15]}$ oder Aurora-Kinasen ${ }^{[13,16]}$ demonstrieren, dass niedermolekulare Verbindungen nicht nur wertvolle Sonden für die Grundlagenforschung sind, sondern auch neue Möglichkeiten für die Behandlung mitosebedingter Krankheiten wie Krebs eröffnen. Die Analyse der zellulären Effekte von BTB-1 ist zurzeit in Arbeit.

Stichwörter: Antiproliferation - Inhibitoren - Enzyme · Mitose · Molekulare Motoren

[1] T. Wittmann, A. Hyman, A. Desai, Nat. Cell Biol. 2001, 3, E28.

[2] M. I. Mayr, S. Hummer, J. Bormann, T. Gruner, S. Adio, G. Woehlke, T. U. Mayer, Curr. Biol. 2007, 17, 488.

[3] M. L. Gupta, Jr., P. Carvalho, D. M. Roof, D. Pellman, Nat. Cell Biol. 2006, 8, 913.

[4] V. Varga, J. Helenius, K. Tanaka, A. A. Hyman, T. U. Tanaka, J. Howard, Nat. Cell Biol. 2006, 8, 957.

[5] T. U. Mayer, T. M. Kapoor, S. J. Haggarty, R. W. King, S. L. Schreiber, T. J. Mitchison, Science 1999, 286, 971.

[6] Z. Maliga, T. M. Kapoor, T. J. Mitchison, Chem. Biol. 2002, 9, 989.

[7] J. Stumpff, G. von Dassow, M. Wagenbach, C. Asbury, L. Wordeman, Dev. Cell 2008, 14, 252.

[8] S. DeBonis, D. A. Skoufias, L. Lebeau, R. Lopez, G. Robin, R. L. Margolis, R. H. Wade, F. Kozielski, Mol. Cancer Ther. 2004, 3 , 1079.

[9] S. Hotha, J. C. Yarrow, J. G. Yang, S. Garrett, K. V. Renduchintala, T. U. Mayer, T. M. Kapoor, Angew. Chem. 2003, 115, 2481; Angew. Chem. Int. Ed. 2003, 42, 2379.

[10] N. Sunder-Plassmann, V. Sarli, M. Gartner, M. Utz, J. Seiler, S. Huemmer, T. U. Mayer, T. Surrey, A. Giannis, Bioorg. Med. Chem. 2005, 13, 6094.

[11] A. F. Straight, A. Cheung, J. Limouze, I. Chen, N. J. Westwood, J. R. Sellers, T. J. Mitchison, Science 2003, 299, 1743.

[12] P. Lénárt, M. Petronczki, M. Steegmaier, B. Di Fiore, J. J. Lipp, M. Hoffmann, W. J. Rettig, N. Kraut, J. M. Peters, Curr. Biol. 2007, 17, 304.

[13] U. Peters, J. Cherian, J. H. Kim, B. H. Kwok, T. M. Kapoor, Nat. Chem. Biol. 2006, 2, 618.

[14] W. Reindl, J. Yuan, A. Kramer, K. Strebhardt, T. Berg, Chem. Biol. 2008, 15, 459.

[15] A. Santamaria, R. Neef, U. Eberspacher, K. Eis, M. Husemann, D. Mumberg, S. Prechtl, V. Schulze, G. Siemeister, L. Wortmann, F. A. Barr, E. A. Nigg, Mol. Biol. Cell 2007, 18, 4024.

[16] C. Ditchfield, V. L. Johnson, A. Tighe, R. Ellston, C. Haworth, T. Johnson, A. Mortlock, N. Keen, S. S. Taylor, J. Cell Biol. 2003, $161,267$. 